\title{
Combinational Optimization of Camping Scheduling
}

\author{
Songsong Wang \\ Shandong University of \\ Science and Technology, \\ Qingdao 266590, China
}

\author{
Xiaowen Ji \\ Shandong University of \\ Science and Technology, \\ Qingdao 266590, China
}

\author{
Cong Liu* \\ Shandong University of \\ Science and Technology, \\ Qingdao 266590, China
}

\begin{abstract}
With the rise in popularity of river rafting, the park managers are faced with the problem of allowing more trips to travel down the river and providing wilderness experience for the visitors, with minimal contact with other groups of boats on the river as well. In this paper, the schedule of trips on the Big Long River (225 miles) is studied. The drifting vessels that can be chosen are oarpowered rubber rafts and motorized boats. By using the queuing theory, we propose several schedules, including the departure time, arriving time, duration (measured in nights on the river) and propulsion (motor or oar), based on some basic and practical assumptions. All schedules proposed here can provide visitors with a wilderness experience, with no two sets of campers occupying the same site at the same time. Based on the number of campsites, the transportation tool, the days of trip and some reasonable assumption, the paper model the above optimal schedule problem as a combinational optimization problem. We use heuristic algorithm to solve the above combinational optimization problem. First of all, we build the matrix which representing arrangement of a boat in 180 days. Secondly, we build the matrix which means the arrangement of campsites in one day. Third, we choose matrix which meet the constraint condition and have the maximized cardinal of line. The number of maximized cardinal of line is the optimal solution of the original combinational optimization problem. So we can see that the result is enormous, According to this model, we can get two-dimensional matrix, it will Simplify the results. The maximum of quantity of matrix which satisfy the conditions is optimal solution.
\end{abstract}

Keywords: matrix permutation and combination; Capacity of the river

\section{DESCRIPTION}

In this subject, the model is based on how to use optimized model to solve the boats of dock problem. We use the combinatorial optimization method [1], [2]. In this method, we suppose matrix to imitate the boats of dispatch. According to matrix addition to imitate the situation of camps. The daily situation of boats starting off can make up a two-dimensional matrix. Through a series of constraint conditions, we can optimize the result step by step.

There are Y camp sites on the Big Long River, distributed fairly uniformly throughout the river corridor. Given the rise in popularity of river rafting, the park managers have been asked to allow more trips to travel down the river. They want to determine how they might schedule an optimal mix of trips, of varying duration (measured in nights on the river) and propulsion (motor or oar) that will utilize the campsites in the best way possible. We also take many practical concerns into account. We discuss the influence of uncertain things on the trip that our algorithm is most useful in reduce the errors.

\section{THE PROBLEM ANALYSIS}

Our task is to design a model to calculate the number of the boats. Train of the thought: The opening days is 180 days. One boat need 6-18days, which is continuous, to pass through the river. So we should remove 6-18days continuous from the 180 days. And we can use a matrix M to express this situation. That is forming a matrix which includes 180 rows and Y columns. In every row only one element is 1 representing the campsite, with others is 0 representing that there is no campsite.

The biggest headaches are

(1) How to arrange boats to make the $\mathrm{X}$ is maximal.

(2) How to calculate the number of boats everyday.

(3) How to choose boats and how many boats should be sent in one day,

(4) How to select the campsites and satisfied the constraint condition.

First of all, we should build a matrix to show arrangement of a boat in 180 days. Secondly, we should build a matrix to show arrangement of campsites in one day. Third, we should 
choose matrix which meet the constraint condition and to do permutations and combinations. Also, we must be clear that we have constraints, which include some factors, such as time, the distance between two campsites adjacent, the speed of the boat. So we should exclude some unsuited plans.

\section{ASSUMPTIONS}

Please use a 9-point Times Roman font, or other Roman font with serifs, as close as possible in appearance to Times Roman in which these guidelines have been set. The goal is to have a 9-point text, as you see here. Please use sans-serif or non-proportional fonts only for special purposes, such as distinguishing source code text. If Times Roman is not available, try the font named Computer Modern Roman. On a Macintosh, use the font named Times. Right margins should be justified, not ragged.

(1) A boat can stay at a campsite but once a day. Because the government agency responsible for managing this river wants every trip to enjoy a wilderness experience, with minimal contact with other groups of boats on the river. The government agency responsible for managing this river wants every trip to enjoy a wilderness experience, with minimal contact with other groups of boats on the river.

(2) All the boats have the same departure time in the morning, doing this we can make sure that when the later boat arrive at the campsite the campsite is empty.

(3) We should suppose the weather of everyday is good, so each boat can travel at its appointed speed.

(4) The number of starting boats (or landing boats) is affected by one factor, which is the number of campsites.

(5) The time of starting boat and stopping time is ignored, only in this way, can we make the model more simple.

(6) Every campsite can hold only one boat at the same time, considering that the campsites is not big enough to hold more than one boat.

(7) The boats must be at the campsites before 6:00 pm. Because it is safer for visitors to stay at campsites at night. And visitors can have a rest and prepare equipment for tomorrow. What's more, the river is nature and valuable, we should not use it frequently.

(8) Every boats have their own independence at any time. so visitors can enjoy themselves.
(9) The days every boats stay at one campsite can not exceed one.

(10) Every trip can not change its transportation during the journey. Because managers have make the schedule, so visitors need to make an appointment on the phone.

\section{MODEL}

Suppose the number of campsites is $Y$, and number the campsites as $1,2, \ldots, Y$ along the river from upstream to downstream. We can use a $Y$-dimension vector to denote the camping arrangement of a group at some night on the travel, the element of vector is 0 or 1 , for example $(1,0,0, \ldots 0)$ represent this group will camp at campsite 1 . If the element of the vector is 0 , this means that the group does not start or have finished his travel. So we can use a matrix $M_{i}$ represent a travel group $i$ travel arrangement during a six month period, which is a $180 \times|Y|$ matrix, the row vector is the camp arrangement in some night. Suppose the set of feasible travel arrangement is $L=\{1,2, \ldots, n\}$. Because two sets of campers can occupy the same site at the same time. This means that we should find a subset of $L$ such that two sets of campers can occupy the same site at the same time. On the other hand, the river managers want to utilize the campsites in the best way possible. This means the cardinal number of the subset must be maximized. So we can model the river manager's problem as an optimization problem as follows:

$$
\begin{aligned}
& \max |U| \\
& \text { s.t. } \\
& \left\{\begin{array}{r}
U=\left\{i_{1}, \cdots, i_{m}\right\} \in P(L), m=1, \cdots, n \\
\left(M_{i_{1}}, \cdots, M_{i_{m}}\right)\left(\begin{array}{c}
1 \\
\vdots \\
1
\end{array}\right) \leq\left(\begin{array}{c}
(1, \cdots, 1) \\
\vdots \\
(1, \cdots, 1)
\end{array}\right)
\end{array}\right.
\end{aligned}
$$

In the ideal case, all the boats fully comply with the arrangement of makers. Campsite can be built everywhere along the river which is 225 miles long. So the campsites can be distributed fairly uniformly throughout the river corridor.

Suppose the number of campsites is $Y, Z_{i}$ represent the $\mathrm{i}$ campsites.

$$
z_{i}=\left\{\begin{array}{l}
1 \\
0 \quad(1 \leq i \leq Y, \quad \mathrm{i} \in \mathrm{N})
\end{array}\right.
$$

Here 1 represents that the boats will call in at $i$, and 0 represents that the boat does not stop at the place. Then this case can be expressed by a one-dimensional vector $\mathrm{T}$. 


$$
T=\left\{z_{1}, z_{2}, \cdots, z_{Y}\right\}
$$

The daily situation of boats starting off can make up a two-

dimensional matrix $A=\left(a_{i j}\right)_{m \times n\left(a_{i j}=0 \text { or } 1\right)}$

$a_{i j}$ represents the situation of the $\mathrm{i}$ boat stopping at the $\mathrm{j}$ campsite.

Suppose $\mathrm{p}$ represents a one-dimensional vector with all of its element is 1 .And the arrangement of the boats must sunder this constraints

$$
\begin{aligned}
& \sum_{i=1}^{m} p a_{i 1} \leq 1 \\
& \sum_{i=1}^{m} p a_{i 2} \leq 1 \\
& \sum_{i=1}^{m} p a_{i n} \leq 1
\end{aligned}
$$

Meanwhile, the daily number of boats starting off should be record.

The arrangement of boats ought to make the utilization ratio of campsites as high as possible and reach the highest number of boats starting off in this 180 days.

The model should be built when the target of system is the best.

$$
\max X=\sum_{m=1}^{180} x_{m}
$$

$X m$ represents the daily number of boats setting off the original.

Overall optimization;

Train of the thought: The opening days is 180days.One boat need 6-18days, which is continuous, to pass through the river. So we should remove 6-18days continuous from the 180 days. And we can use a matrix $M$ to express this

\section{SOLUTION AND RESULT}

(1) The first step: Supposing the value of $Y$

If we don't assign a value to $\mathrm{Y}$ and we don't optimize our choosing, we will have a great many of results. situation. That is forming a matrix which includes 180 rows and $\mathrm{Y}$ columns .In every row only one element is 1 representing the campsite, with others is 0 representing that there is no campsite. Then using matrix multiplication or matrix logic exclusive OR (XOR) to express the constraint situation. Everyday the boats can not stay at the same campsite. First, we should list all the matrix (m) satisfying the constraints. Then we combine this matrix $(m)$ satisfying the constraints. The number of $\mathrm{m}$ in the combination which has the most number of matrix is $\mathrm{x}$ which is what we need.

Such as:

$$
\begin{gathered}
\left(M_{i j}\right)_{180 \times Y}=\left[\begin{array}{ccccc}
0 & 0 & 0 & \ldots & 0 \\
0 & 0 & 0 & \ldots & 0 \\
0 & 1 & 0 & \ldots & 0 \\
0 & 0 & 1 & \ldots & 0 \\
0 & 0 & 0 & 0 & 1
\end{array}\right\} \\
\sum_{j=1}^{Y} M_{i j} \leq 1 \\
\quad(1<=\mathrm{i}<=180)
\end{gathered}
$$

that is every row has only 1 or no 1 .

The 6-8 days continuous which are selected from the 180 days make up the combinations. And the selection of the first campsite is in a certain range.

Supposing that the first campsite of first day the boat stop at is the $\mathrm{j}$ campsite and the boats move ahead as fast as possible

$$
1<=\mathrm{j}<(8 \times 12) \div(225 \div Y)
$$

And the next situation of 1 should be bigger than the value of j. $M_{t}$ is the situation the j boat select.

Provided $N_{1 t}=\left\{\begin{array}{c}M_{1} \\ M_{2} \\ M_{3} \\ \ldots \\ M_{t}\end{array}\right\}$

Per element of the one-dimensional vector represent the situation of arrangement. Supposing that there L answers which may solve the problem.

The best answer $\mathrm{N} \in \mathrm{L}$.

From the result of software, when $\mathrm{Y}=18$ we can get different plans of stopping the boats just as the days on the trip is 6.Here we assume all the boats are fast boats. 
International Journal of Science and Engineering Applications

Volume 5 Issue 4, 2016, ISSN-2319-7560 (Online)

Table 2: the whole boats' camp arrange

\begin{tabular}{|c|c|c|c|c|c|c|c|c|c|}
\hline $\begin{array}{c}\text { Groups } \\
\text { days }\end{array}$ & $\mathbf{1}$ & $\mathbf{2}$ & $\mathbf{3}$ & $\mathbf{4}$ & $\mathbf{5}$ & $\mathbf{6}$ & $\mathbf{7}$ & $\ldots . . . .$. & $\mathbf{1 8 0}$ \\
\hline 1 & 1 & 1 & 1 & 1 & 1 & 1 & 0 & 00000 & 0 \\
\hline 2 & 0 & 1 & 1 & 1 & 1 & 1 & 1 & 00000 & 0 \\
\hline 3 & 0 & 0 & 1 & 1 & 1 & 1 & 1 & 10000 & 0 \\
\hline 4 & 0 & 0 & 0 & 1 & 1 & 1 & 1 & 1000 & 0 \\
\hline$\ldots .$. & $\cdots$ &.. &.$\cdot$ &.. &.$\cdot$ & $\cdots$ &.$\cdot$ &.. &.. \\
\hline
\end{tabular}

The situation of the boats setting off per day according to one of the groups is as follows:

The average distance the boats travel is 37.5 miles per day, the average time the boats travel is 4.6875 hours. It will take 1.5625 hours to run across the two campsites which are adjacent. Everyday one boat can travel as many as 12 hours ,that is 96 miles. Such as: When a boat pass across 7 campsites a day, we have to select 5 campsites from the last 11 campsites.

For example :1-7,2-8,3-9,4-10,5-11,6-12,7-13, 8-14,9-15,10-

$16,11-17,12-18$

$M_{1}=\{111111000000010000000\}$

Table 3: different date of percentage

\begin{tabular}{|c|c|c|}
\hline Trip choosing & Time choosing & percentage \\
\hline Short motor trips & $6-8$ days & 22 \\
\hline Long motor trips & 9 or more days & 32 \\
\hline Short oar trips & 14 or fewer days & 26 \\
\hline Long oar trips & 15 or more days & 29 \\
\hline
\end{tabular}

In order to directly received through the senses, we draw the pie chart as follows.

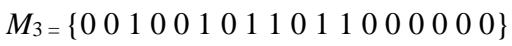

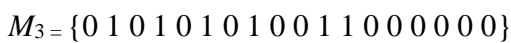

(2) Then get the situations when a boat pass across 6, 5,4,3campsites.

From the information we can know the running conditions of the Big Long River; 


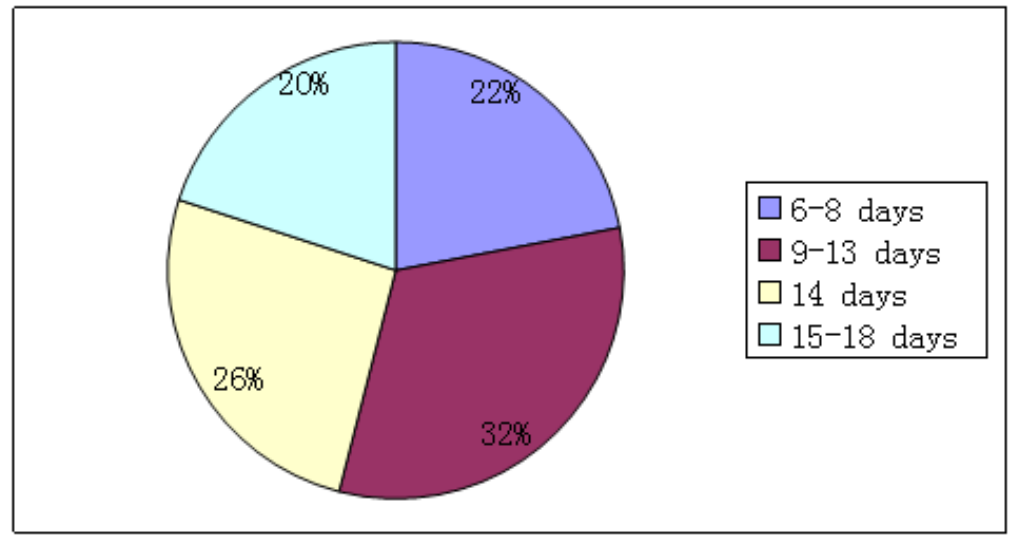

Figure 3:different date of percentage(pie chart)

We can get a method which can distribute the time on average. In order to get full use of the camps, we can fix short-time with long-time.

Table 4: average distribution of boats in every day

\begin{tabular}{|l|l|l|l|l|l|l|l|l|l|l|l|l|l|}
\hline \multicolumn{10}{|c|}{ the distribution of boats in 13 days } \\
\hline one & 6 & 7 & 8 & 9 & 10 & 11 & 12 & 13 & 14 & 15 & 16 & 17 & 18 \\
\hline two & 18 & 17 & 16 & 15 & 14 & 13 & 12 & 11 & 10 & 9 & 8 & 7 & 6 \\
\hline three & 6 & 7 & 8 & 9 & 10 & 11 & 12 & 13 & 14 & 15 & 16 & 17 & 18 \\
\hline four & 15 & 14 & 13 & 12 & 11 & 10 & 9 & 8 & 7 & 6 & & & \\
\hline sum & 45 & 45 & 45 & 45 & 45 & 45 & 45 & 45 & 45 & 45 & 40 & 41 & 42 \\
\hline
\end{tabular}

\section{ANALYSIS OF RESULT}

$Y=45$,there are 13 different plans for boats

If we don't think about the constraint condition, we will have many results. For example:

00000001000000010000000100000001000000010000 00000010000001000000100000010000001000000100 00000100000100000100000100000100000100000100 00000100000100000100000100000100000100000100 00001000010000100001000010000100001000010000 00001000010000100001000010000100001000010000 00010001000100010001000100010001000100010001 00010001000100010001000100010001000100010001 00010001000100010001000100010001000100010001 00010001000100010001000100010001000100010001 00100100100100100100100100100100100100100100 
So we can see that the result is enormous.

In local optimum, we use a two dimension matrix to express the situation of boats every day. So we can get the max number of combination as the max quantity of boats. And by this analogy, we can calculate the sum of the boats in 180 days. In the global optimization, we use matrix $M$ to signify driving conditions in 180 days. The element of the matrix

\section{BACKGROUND}

In nowadays more people like to travel and meantime the river trips is very popular in recent years. Because river trips is an adventure sports. Visitors to the Big Long River (225 miles) can enjoy scenic views and exciting white water rapids. The river is inaccessible to hikers, so the only way to enjoy it is to take a river trip that requires several show the situation of boats. The maximum of quantity of matrix which satisfy the conditions is optimal solution. Because of the time, in the solving of the model, we only deal with one situation ,and other situations are similar with the situation. In order to make the computing more simple, we set $\mathrm{Y}$ as 18 .Meanwhile, all the boats are the fast boats and all the boats need 6 days to pass across the river.

days of camping. In order to make the journey more safe we have to build some campsites along the river.

We know that the trip involves so many factors that we can not calculate the accurate the sum of quantity of boats in one year, so we ought to simplify the travel. In order to make more boat trips be added to the Big Long River's rafting season, we design an optimized model to solve this problem.

Table 1: Popular attraction sites in the river corridor during the typical flow regimes of 1998-2000(sheet)

\begin{tabular}{rlrl}
\hline River mile & Site name & Rank & Frequency \\
\hline 136.2 & Deer Creek & 1 & 0.91 \\
156.8 & Havasu & 2 & 0.88 \\
32.8 & Redwall & 3 & 0.82 \\
116.5 & Elves Chasm & 4 & 0.77 \\
61.4 & Little Colorado & 5 & 0.66 \\
87.8 & Phantom & 6 & 0.61 \\
108.7 & Shinumo & 7 & 0.56 \\
147.9 & Matkatamiba & 8 & 0.48 \\
132.0 & Stone CK & 9 & 0.41 \\
47.2 & UPR Saddle & 10 & 0.34 \\
31.6 & South Canyon & 11 & 0.31 \\
53.0 & Nankoweap & 12 & 0.3 \\
84.0 & Clear CK & 13 & 0.28 \\
34.8 & Nautiloid & 14 & 0.26 \\
133.8 & Tapeats & 15 & 0.25 \\
\hline
\end{tabular}

In order to directly run over the senses, we draw the histogram as follows. 


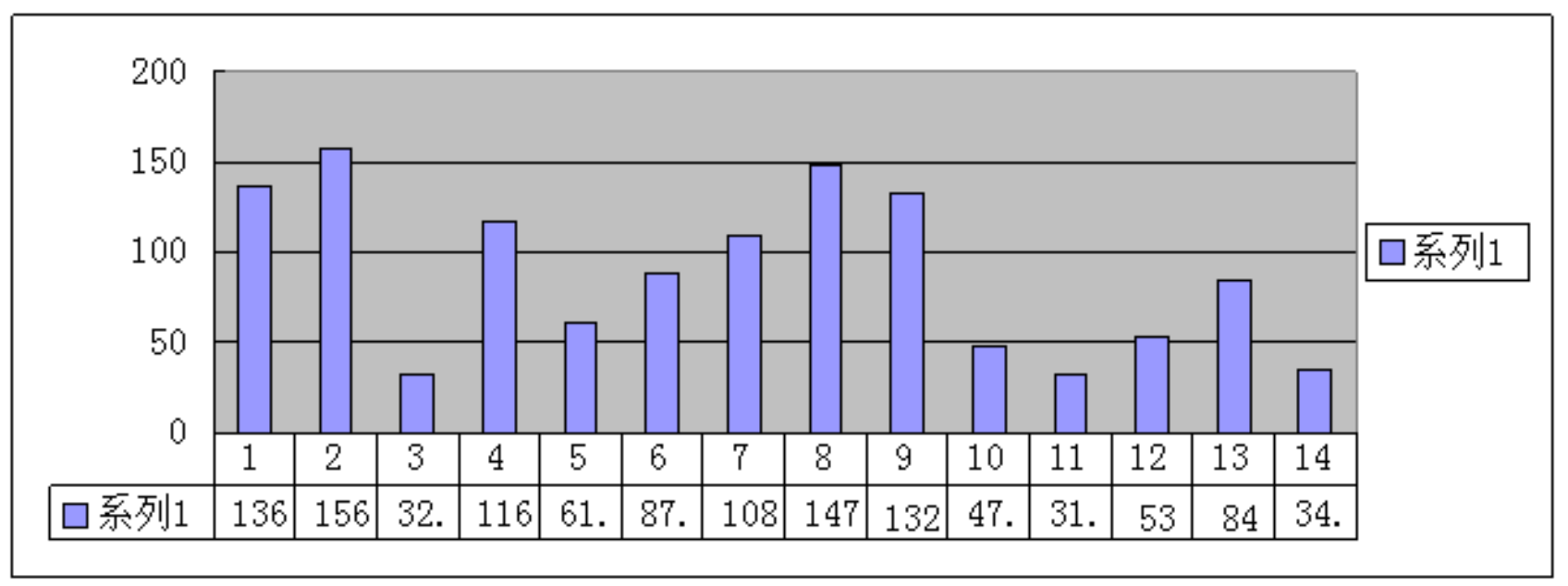

Figure 1: Popular attraction sites in the river corridor during the typical flow regimes of 1998-2000(histogram)

So we can know the generally range of $\mathrm{Y}$ from the chart above. When we do model analysis and mode solution, we will have a reasonable assumption. When we figure out one of the $\mathrm{X}$, we will compare with the quantity of the camps above. If the result of assumption is far away from it. we will adopt another one.

The dates can regards as one part of one row of matrix $M_{\mathrm{ij}}$. The general methods to solve the combinatorial optimization problems of $0-1$ are method of exhaustion and implicit enumeration. The method of exhaustion need to test all the solutions , provided the model has $\mathrm{K}$ variables, then we need to examine $2^{Y}$ times, the calculated amount is very big. The implicit enumeration impliedly search all the solutions, and this method can be divided into Backtracking Algorithm ,Branch and Bound method, and Sequential Combination Tree Algorithm, in this way, we can reduce the calculated amount, and find the best answer. According to the feature of this model ,this article determine the search range by the methods of solution space decomposition and of solution space limit method, and seek the best answer by the method of Sequential Combination Tree Algorithm. From the picture, we divide the solution space into several subspaces according to certain rules. And confine the subspaces which are infeasible and which are feasible but are not the best subspaces, so as to narrow down the search range. In the new search range, we can calculate the best answer exactly.

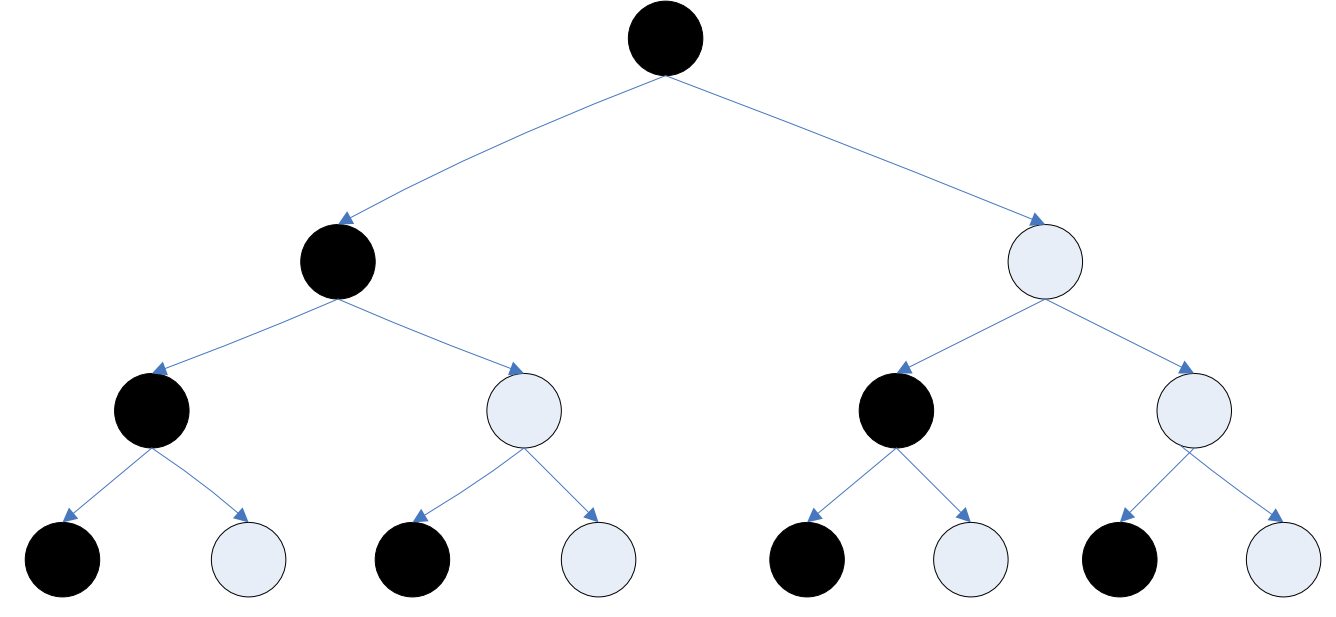

Figure 4:simulation of choosing in every day(the black round show the camp is full, the white round show the camp is empty)

The black dots represent 1 , the white dots represent 0 ,and the layer where the dots locate represents the situation of the campsite .There are Y layers. Supposing the days the sailing time of the boats is d days. $6 \leqslant d \leqslant 18$ then $\sum_{i=1}^{Y} z_{i}=d$.

\section{ADVANTAGE AND DISADVANTAGE}

The structure of Heuristic algorithm is based on intuitive judgment or experience. We can get a feasible solution of the problem in the condition of acceptable cost (time cost, space cost etc.)The rate of deviation between the solution that we get and the optimum solution can not be predicted. We can get the best solution under the acceptable conditions. but we can not guarantee the solution is the optimal solution and it might be infeasible. Even in most cases. we can not point out the approximate between the optimal solution and the solution we get.

Although we not always can get optimal solution ,but we can get the solution most close to the optimal solution.

Heuristic supply low bifurcate rate for each to solve specific problems of search trees each node, so they have better efficiency of computing .It is easier to get reasonable solution. Comparing these solution to choose the solution as optimum solutions. 
Our model effectively achieved all of the goals we set initially. It was fast and could handle large quantities of data, but also had the flexibility we desired. Though we did not test all possibilities, we showed that our model optimizes state districts for any of a number of variables. If we had chosen to input more income, we could have produced high-quality results with virtually no added difficulty. As well, our method was robust.

\section{CONCLUSIONS}

During the process we realized that teamwork is very important in our daily life, especially when the mission can not be handled. by only one person. Before action we should divide the job into different parts .Also we need to make sure that all of the members of the team can have his own duty .Only every one in the team make his great effort, can the team achieve great grades. We should consider every problem from all the aspects. In the process we should collect a mass of material ,so effective information search is of very important. As to the materials collected, it is vital to refine the information, which is useful for us. In the future, we prefer to integrate the optimization with some traditional data mining approaches [3], [4], [5] to find more effective ways for optimization.

\section{REFERENCES}

[1] Sherali H D, Desai J, Rakha H. A discrete optimization approach for locating automatic vehicle identification readers for the provision of roadway travel times[J]. Transportation Research Part B: Methodological, 2006, 40(10): 857-871.

[2] Lin X, Shroff N B. Utility maximization for communication networks with multipath routing[J]. IEEE Transactions on Automatic Control, 2006, 51(5): 766-781.

[3] Ting Lu, Qi Gao, Xudong Wang, Cong Liu, "Modified Page Rank Model in Investigation of Criminal Gang", International Journal of Science and Engineering Applications, 4(3): 100-104, 2015.

[4] Baohua Liu, Xudong Wang, Qi Gao, Cong Liu, "A KMeans based Model towards Ebola Virus Prorogation Prediction", International Journal of Science and Engineering Applications, 4(3): 95-99, 2015.

[5] Libao Zhang, Faming Lu, An Liu, Pingping Guo and Cong Liu*, "Application of K-Means Clustering Algorithm for Classification of NBA Guards", International Journal of Science and Engineering Applications, 5(1), pp. 1-6, 2016. 